\title{
LÓGICA CLÁSICA Y ESQUIZOFRENIA: POR UNA SEMÁNTICA LÚDICA
}

\author{
Juan Redmond \\ Instituto de Filosofía, Universidad de Valparaíso, Chile \\ Juan.redmond@usal.es
}

Rodrigo Lopez-Orellana

Instituto de Estudios de la Ciencia y la Tecnología (ECYT)

Universidad de Salamanca, España

rodrigo.lopez@usal.es

\begin{abstract}
RESUMEN / ABSTRACT
En este artículo delineamos una propuesta para elaborar una lógica de las ficciones desde el enfoque lúdico del pragmatismo dialógico. En efecto, centrados en una de las críticas mayores al enfoque clásico de la lógica: la esquizofrenia estructural de su semántica (Lambert 2004: 142-143; 160), recorremos los compromisos ontológicos de las dos tradiciones mayores de la lógica (Aristóteles y Frege) para establecer sus posibilidades y límites en el análisis del discurso ficcional, y la superación desde una perspectiva lúdico pragmática.
\end{abstract}

PALABRAS ClavE: lógica, dialógica, cuadro de oposición, esquizofrenia, semántica lúdica, cuantificador existencial, nombres propios, intuicionismo, tercero excluido, doble negación, ficciones.

\section{Classical Logic and Schizophrenia: For a NeUtral Game Semantics}

In this paper we draw a proposal to develop a logic of fictions in the game-theoretical approach of dialogical pragmatism. From one of the main criticisms that point to classical logic: the structural schizophrenia of its semantics (Lambert, 2004: 142-143; 160), we review the ontological commitments of the two main traditions of logic (Aristotle and Frege) to highlight their limits concerning the analysis of fictional discourse, and the overcoming from a pragmatic game perspective.

KEYWORDS: Logic, dialogic, traditional square of opposition, schizophrenia, game semantics, existential quantification, proper names, intuitionism, principle of excluded middle, double negation rule, fictions. 


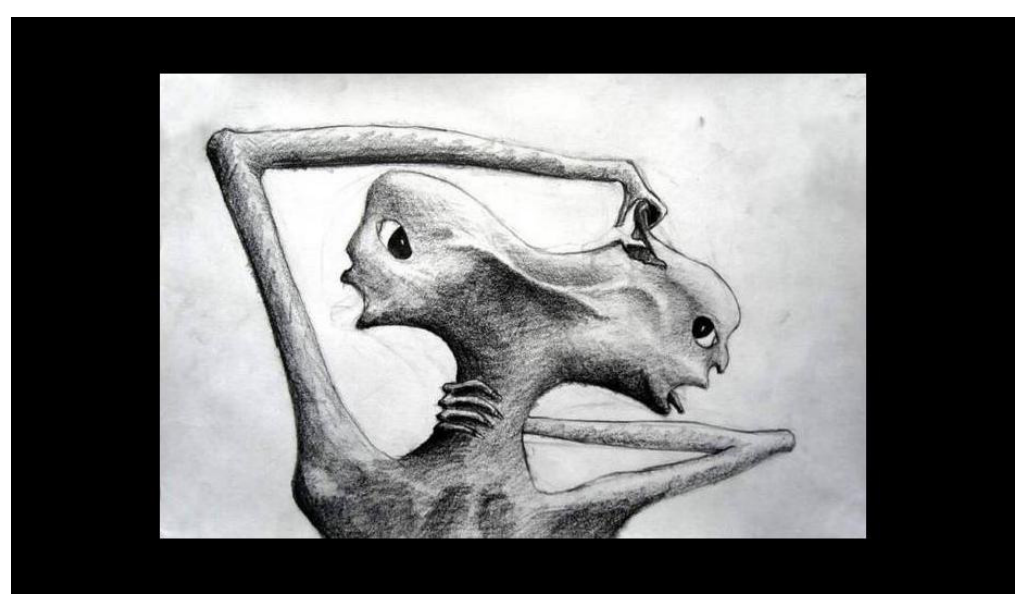

\section{Aristóteles y el Cuadrado de Oposición}

RR

El Cuadrado de Oposición de Aristóteles nos enseña que si la oración Todo $S$ es $P^{1}$ (universal afirmativa) es verdadera, la universal negativa correspondiente (Ningún $S$ es $P$ ) no puede ser verdadera al mismo tiempo. A esta relación la llamó "contraria", es decir, ambas pueden ser falsas al mismo tiempo pero no verdaderas. Las particulares correspondientes también poseen una relación específica y se llama “subcontrariedad". Es decir, Algún S es $P$ y Algún S no es $P$ pueden ser verdaderas al mismo tiempo pero no falsas al mismo tiempo. De manera cruzada se establece una relación que llamó contradictoria: si una es verdadera, la otra debe ser falsa y viceversa. Finalmente tenemos la relación de "subalternación" entre la universal (afirmativa o negativa) y la correspondiente particular. Cabe notar que si bien Aristóteles consideraba las oraciones singulares del tipo "Sócrates es ateniense" (recordemos que en las anteriores $S$ y $P$ son conceptos y aquí Sócrates es un nombre propio), no hacen parte de los silogismos tal y como son capturados por los modos y las figuras.

1 Más abajo diremos como Frege: “oraciones aseverativas” (Behauptungsätze). 
Aquí el cuadrado:

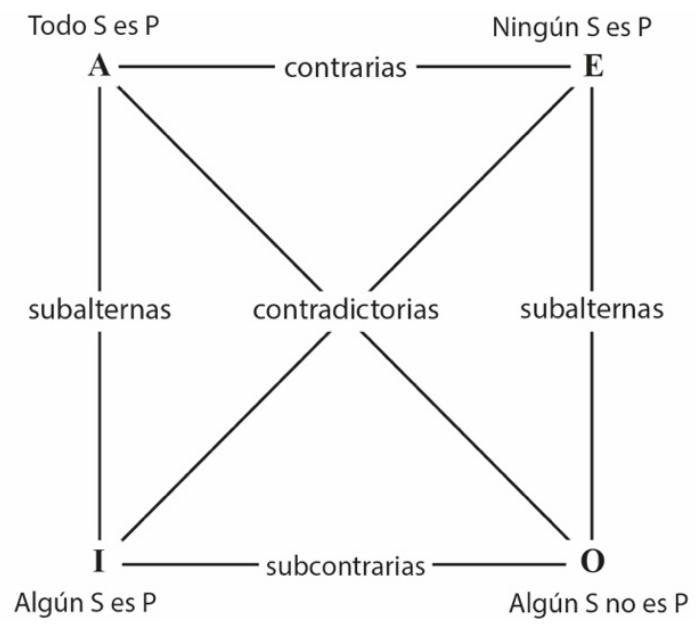

\section{Origen del Cuadrado de Oposición}

Este Cuadrado no fue expuesto de manera integral por Aristóteles (Parsons 2012). En efecto, Aristóteles solo enuncia una parte de las relaciones y la referencia principal corresponde a De Interpretatione 6-7, que contiene tres afirmaciones: que A y O son contradictorias, que E e I son contradictorias, y que A y E son contrarias (17b, 17-26):

Digo, pues, que se opone contradictoriamente a la negación la afirmación que significa lo universal respecto a lo mismo que $<$ la negación significa $>$ de manera no universal, v.g.: $\langle e s\rangle$ todo hombre blanco - no $\langle e s\rangle$ todo hombre blanco, $<$ no es $>$ ningún hombre blanco - es algún hombre blanco; [20] <se oponen> contrariamente la afirmación de lo universal y la negación de lo universal $<$ como tales>, v.g.: $<e s>$ todo hombre justo $-<$ no es $>$ ningún hombre justo; por lo tanto estas últimas no pueden ser simultáneamente verdaderas, mientras que las opuestas a ellas cabe <que lo sean> en relación con la misma cosa, v.g.: no $<e s>$ todo hombre blanco y es algún hombre blanco.. (Aristóteles 1995: 46) ${ }^{2}$

2 "I call an affirmation and a negation contradictory opposites when what one signifies universally the other signifies not universally, e.g. every man is white -not every man is white, no man is white - some man is white. But I call the universal affirmation and the universal negation contrary opposites, e.g. every man is just-no man is just. So these cannot be true together, but their opposites may both be true with respect to the same thing, e.g. not every man is white -some man is white". (Siguiendo la traducción y notas de J. L. Ackrill (1963), Aristotle Categories and De Interpretatione: 48 [17b16]). 
Esto se esquematiza así:

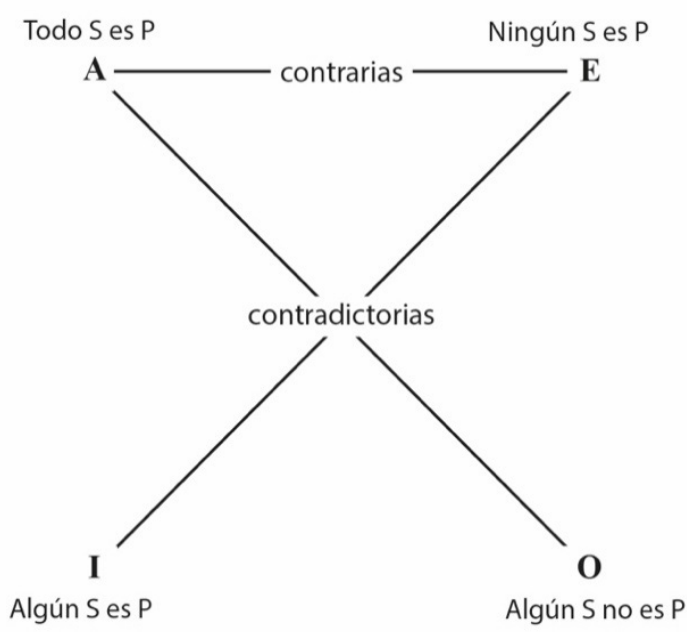

Como bien señala Terence Parsons en su artículo de la Enciclopedia Stanford, el resto se obtiene por implicación. Por ejemplo, podemos mostrar que I y $\mathrm{O}$ no pueden ser ambas falsas pero si verdaderas (subcontrarias): si I es falsa, E es verdadera (contradictoria), A es falsa (contraria de E) y por ende O debe ser verdadera. Del mismo modo para la subalternación. Por ejemplo: si A es verdadera, $\mathrm{O}$ es falsa (contradictoria) y por ende I es verdadera (subcontrarias).

\section{Relectura del Cuadrado de Oposición desde la lógica clásica}

Dicho de manera directa, la lógica clásica disuelve el Cuadrado de Oposición. En efecto, pasados más de dos mil años de inmutabilidad del cuadro de oposición, la lógica clásica -sobre sus fundamentos- se permite una relectura del cuadro de oposición que lo reduce solo a sus relaciones "contradictorias". Las restantes desaparecen. Profundizaremos en este punto.

Llamamos lógica clásica a la perspectiva de la lógica que se despliega de modo exponencial a partir, principalmente, de la obra de Gottlob Frege. Este despliegue fructífero se debe, entre otras genialidades de este matemático, a su interpretación de los cuantificadores como operadores lógicos.

Las oraciones de Aristóteles son traducidas al nuevo lenguaje del siguiente modo:

$\begin{array}{ll}\text { Todo } S \text { es } P & \forall \mathrm{x}(S x \rightarrow P x) \\ \text { Ningún } S \text { es } P & \forall \mathrm{x}(S x \rightarrow \neg P x) \\ \text { Algún } S \text { es } P & \exists \mathrm{x}(S x \& P x) \\ \text { Algún } S \text { no es } P & \exists \mathrm{x}(S x \& \neg P x)\end{array}$


Si elegimos un predicado $S x$ para el cual toda afirmación sea falsa, se puede demostrar fácilmente que el cuadro queda reducido a lo siguiente:

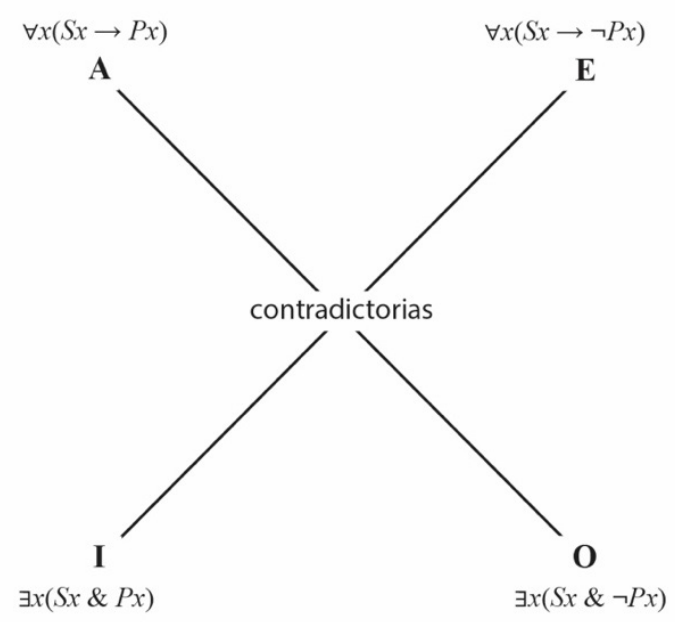

No olvidemos que esto corresponde al lenguaje estandarizado a partir de los trabajos de Bertrand Russell que tuvo en cuenta el lenguaje utilizado por Giuseppe Peano. Pero en el original de Frege de Begriffsschrift de $1879^{3}$ reza así:

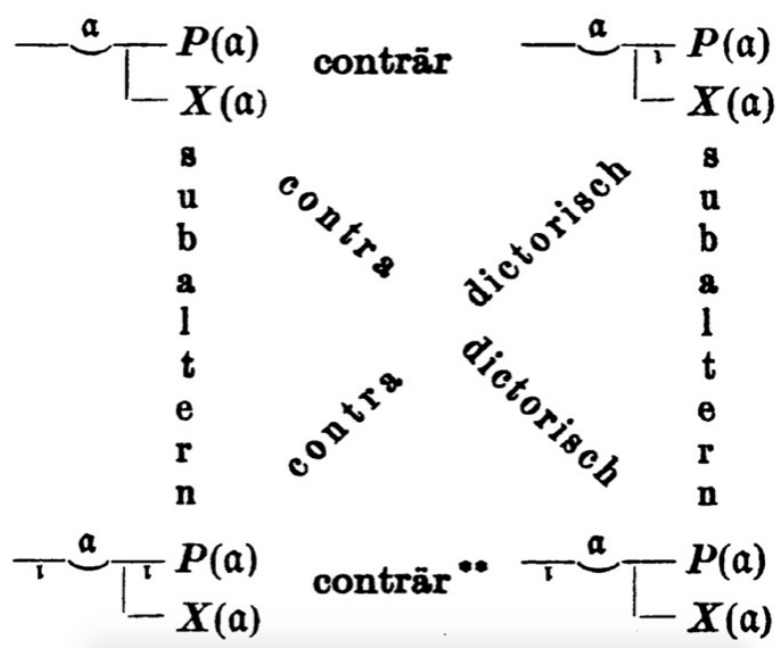

Aquí en la versión de Ignacio Angelelli de 1964 (Frege 1993: 24). 
En nuestro artículo no nos ocuparemos del debate en torno a si esta lectura del Cuadrado de Oposición por parte de la lógica clásica es correcta o no. Nos focalizaremos, por el contrario, en los fundamentos sobre los cuales está edificada la semántica de la lógica clásica. Particularmente la semántica de términos generales y singulares. Y específicamente a la posibilidad, mencionada pocas líneas más arriba, de poder considerar un predicado $S x$ para el cual todas las afirmaciones sean falsas.

En efecto, uno de los temas centrales de este artículo es el siguiente: ¿Es posible que Aristóteles no considerara este tipo de predicados (para los cuales toda afirmación es falsa) y la lógica clásica sí? Pero, ¿qué es un predicado para el cual toda afirmación es falsa? Por decirlo de manera breve - pero por analogía-, es un predicado vacio. Es decir, un predicado que no se aplica a ningún individuo, sujeto u objeto: un predicado que no se aplica a nada o que no se puede predicar de nada. La pregunta que no intentaremos responder aquí es si realmente Aristóteles consideró o no este tipo de predicados, aunque claramente parece haber hablado de cosas que no existen. En efecto, menciona un animal fabuloso que ya aparece en Aristófanes (Las ranas) y en Platón (La República 488a): el ciervo-cabrío ${ }^{4}$. Menos aún, saber cómo fue posible que pasaran tantos siglos sin mayores cuestionamientos. Solo daremos algunas pautas. Lo que intentaremos analizar son los alcances de esta posibilidad -la de los predicados vacíos- dentro de la lógica clásica y su consecuencia más notoria y que da el título al presente artículo y que justificarían una lógica libre.

\section{Predicados vacíos y compromiso ontológico: algunas anticipaciones críticas}

De los muchos autores que analizaron y cuestionaron los compromisos ontológicos asumidos por quienes sostienen el Cuadrado de Oposición de Aristóteles, nos detendremos brevemente en Pedro Abelardo (1079-1142). En efecto, este sorprendente lógico ya había notado que se presentaban problemas al momento de considerar que los nombres o los conceptos de las oraciones del Cuadrado de Oposición no se aplicaban a nada. Esto lo señala muy bien De Rijk en su introducción a la Dialéctica de Abelardo (De Rijk 1956: XLIII): para Abelardo, la universal afirmativa 'omnis homo est homo' es falsa si resulta que no hay ningún hombre. Es decir, si no hay ninguna cosa a la cual se aplique el predicado homo ${ }^{5}$. De Rijk se refiere a este parágrafo donde Abelardo se refiere también a las particulares [nuestra traducción]: "De hecho, lo que no se dice

$4 \quad$ “Además, ¿cómo se demostrará el qué es? Pues es necesario que el que sabe qué es el hombre, o cualquier otra cosa, sepa también qué es (en efecto, lo que no es no sabe nadie qué es, aunque el enunciado o el nombre signifiquen algo, <como $>$ cuando digo ciervocabrio, pero es imposible saber qué es un ciervo-cabrío)". (Aristóteles 1995: 405-406).

"Abailard here says, speaking about the opposition of propositions, that the proposition 'omnis homo est homo' is false, if no man exists, i. e. if the class of the bomines is void". 
[que sea verdad], con ningún hombre existente, es que "todo hombre es un hombre" ni que "cierto hombre no es un hombre"(Abelardo, Dial. II, I76 20-21; en De Rijk 1956)

Veamos una explicación sencilla: Algún A es B parece implicar que Algo es A. Por ejemplo, Algún hombre es músico parece implicar que al menos una cosa es un hombre. Es decir, el hombre que tiene que ser músico si es verdad que Algún hombre es músico. ¿Pero qué sucedería -como dice Abelardo en su Dialectica- si no hay ningún hombre? Es decir, si no hay hombres existentes, las oraciones particulares correspondientes serían falsas. Para hacer más claro su punto, Abelardo usa el término 'hombre-piedra' u 'hombre de piedra' [nuestra traducción]:

Si 'Todo hombre-piedra es piedra' es verdadero, también lo es su conversa por accidente: 'algunas piedras son hombre-piedra'. Pero ninguna piedra es un hombrepiedra, porque ni este hombre, ni aquel hombre [y así siguiendo] es una piedra. Pero también es falso por necesidad que 'cierto hombre-piedra no es piedra', puesto que es imposible suponerlo (Abelardo, Logica Ingredientibus, LI 3.07; en De Rijk 1956) ${ }^{7}$.

¿Se le escapó este punto a Aristóteles? Desde luego que no: en sus Categorías [13b] ya había señalado un problema similar, pero para los 'nombres propios' que pudieran no referirse a nada:

[...] en efecto, el que Sócrates esté sano es contrario de que Sócrates esté enfermo; pero tampoco en estos casos es necesario que siempre una de las dos cosas sea verdadera y la otra falsa: pues, existiendo Sócrates, será verdadero lo uno y falso lo otro, pero, no existiendo, ambas cosas serán falsas: en efecto, ni el que Sócrates esté enfermo ni el que esté sano son verdad si, en definitiva, no existe el mismo Sócrates (Aristóteles 1982:70).

Respecto de los términos singulares (nombres propios), la lógica clásica se mantiene fiel a la intuición de Aristóteles, y los desarrollos formales no se hicieron esperar. Los más conocidos son la Teoría de las Descripciones Definidas de Bertrand Russell (Russell 1905) y el mismo Frege que utiliza la clase nula (Frege 1948; Goodman 1949: 3-5). Por razones de espacio no podremos desarrollar estas dos perspectivas aquí.

La lógica clásica acabó con todas las dudas para oraciones universales y particulares, abriendo definitivamente la puerta a los predicados vacíos. Es decir, la lógica clásica considera predicados para los cuales es falsa toda afirmación. Pero con esta franquicia

6 "Re enim hominis prorsus non existente neque ea vera est quae ait: 'omnis homo est homo' nec ea quae proponit: "quidam homo non est homo"'.

"Si enim uera est: 'Omnis homo qui lapis est, est lapis'et eius conuersa per accidens uera est: 'Quidam lapis est homo qui est lapis'. Sed nullus lapis est homo qui est lapis, quia neque hic neque ille etc. Sed et illam: 'Quidam homo qui est lapis, non est lapis'falsam esse necesse est, cum impossibile ponat". 
no solo acabó al mismo tiempo con el Cuadrado de Oposición de Aristóteles, sino que fundó las bases de la lógica clásica sobre un fundamento asimétrico.

\section{De camino a la semántica de la lógica clásica}

Uno de los cambios más drásticos que realizó la lógica clásica fue suprimir el uso de la estructura de sujeto y predicado en el análisis de los argumentos (herencia aristotélica) y su reemplazo por argumento y función. Este cambio trascendental para la lógica, como mencionamos más arriba, se lo debemos a Gottlob Frege. Si bien Edmund Husserl (1859-1938), califica a Frege como un "excéntrico de ingenio agudo pero que ni como matemático ni como filósofo se trataba de alguien originalmente productivo"8 (Gabriel et al. 1976: 92, traducción nuestra), se trata sin duda el artífice de los principales cambios de la nueva era de conocimientos en lógica y filosofía de la lógica.

En efecto, Frege era consciente de que la lógica como disciplina que se ocupa de los razonamientos había llegado a cierto límite de sus potencialidades, y que esto ya había sido notado por otros grandes hombres de la filosofía como Kant:

Lo curioso de la lógica (desde Aristóteles) es que tampoco haya sido capaz, hasta hoy, de avanzar un solo paso. Según todas las apariencias se halla, pues, definitivamente concluida (Kant 1993: 15).

Convencido de la expansión enriquecedora que la lógica tendría a partir de sus descubrimientos, Frege llamó a esta forma de pensar la lógica como "concluida", como "la leyenda de la esterilidad de la lógica pura"9 (Frege 1884: 24; traducción de Stepanians 2007: 3). Desde luego reconoce que hay una suerte de estancamiento, pero cree saber cuál es la causa: la aceptación de ciertas hipótesis de los aristotélicos que él considera erróneas.

En efecto, Frege realiza su primera gran contribución con la publicación de su "librito", como él lo llamaba, el Begriffschrift que es traducido mayormente como Conceptografía. Allí mismo propone la primera ruptura con la tradición aristotélica: "§3. En mi modo de representar un juicio, no tiene lugar una distinción entre sujeto y predicado" (Frege 1993: 2, traducción de Stepanians 2007: 44) ${ }^{10}$. Es decir, el estancamiento se debía -según Frege- a haber creído que argumentamos sobre la base de oraciones estructuradas según la forma de sujeto y predicado.

Contraejemplo al modelo aristotélico:

8 " [...] ein scharfsinniger, aber weder als Mathematiker noch als Philosoph fruchtbringender Sonderling" (Pasaje de carta de Husserl a Heinrich Scholzen 1936).

"[...] und das Märchen von der Unfruchtbarkeit der reinen Logik nicht halten lassen".

"Eine Unterscheidung von Subjekt und Prädicat findet bei meiner Darstellung eines Urteils nicht statt". 
1. En Platea los persas fueron vencidos por los griegos;

2. En Platea los griegos vencieron a los persas.

A pesar de que ambas oraciones tienen distintos sujetos y predicados, son -según Frege- lógicamente equivalentes: lo que se deduce de la primera, también se deduce de la segunda. Es decir, la distinción entre sujeto y predicado no solo no nos ayuda, sino que es un obstáculo al momento de capturar los elementos necesarios para analizar los argumentos.

En su lugar, como mencionamos más arriba, propone una distinción que viene de la aritmética: función y argumento ${ }^{11}$. ¿Qué relación guardan las estructuras aritméticas con los elementos de un lenguaje que usamos para argumentar? Pues, que la primera -según Frege- corresponde a los predicados y la segunda, a los nombres propios. Es decir, para Frege tenemos, por un lado, el nivel sintáctico donde opera la distinción entre nombres propios, predicados, y las oraciones aseverativas (Behauptungssätze) que emergen de ambos. Por otro, el nivel semántico (sus significados [Bedeutung]) que serán respectivamente los objetos y los conceptos (división irreconciliable). En este sentido, como bien señala Stepanians en su preclara introducción a la obra de Frege (Stepanians 2007: 77): "Frege puede reclamar para sí haber desarrollado por primera vez en la historia de la lógica una teoría semántica para un lenguaje y haber puesto así los fundamentos de una teoría general de la validez lógica”.

\section{Estrategias de Frege para expresar generalidad}

Frege muestra la efectividad de su nueva distinción entre argumento y función a través de un nuevo procedimiento para expresar generalidad en el lenguaje, basado en el procedimiento que es similar en aritmética. No olvidemos que Frege era un matemático. La correspondencia que estableció Frege fue la siguiente: A partir de dos expresiones como ‘2 $2+3=5$ ' y 'Juan es músico', si en la primera yo puedo quitar el argumento '2' y obtener la expresión genérica ${ }^{6} x+3=5$ ', en la segunda puedo quitar el nombre propio 'Juan' y obtener de igual modo la expresión genérica 'x es músico'. Ambas expresan generalidad en el sentido siguiente: en ambas expresiones la " $x$ " podría ser reemplazada por cualquier individuo del dominio considerado. De hecho, la " $\mathrm{x}$ " significa que en ese lugar de la oración hay un lugar vacío y que lo puede ocupar cualquier número o nombre propio, respectivamente. Es decir, en la primera por cualquier número, en la segunda por cualquier individuo del universo. Frege llama a esta última expresión -en el marco de sus revolucionarias ideas-como predicado: 'x es músico'. El propio Frege lo expresa con otro ejemplo:

11 El término 'argumento', en este último sentido, es poco feliz para nuestro análisis lógico en castellano. Pero a Frege no debió sonarle mal pues la palabra alemana es Argument que no se confunde con Schluss (inferencia o argumento). 
Mientras que desde el punto de vista aludido antes, "hidrógeno" era el argumento y "ser más liviano que el anhídrido carbónico" era la función, también podemos pensar el mismo contenido conceptual de modo que "anhídrido carbónico" sea el argumento, y "ser más pesado que el hidrogeno" sea la función ${ }^{12}$ (Frege 1879: 15. Nuestra traducción).

Tenemos entonces:

\begin{tabular}{|l|l|l|}
\hline Expresión aritmética & Función & Argumento \\
\hline$' 2+3=5 '$ & $' \mathrm{x}+3=5{ }^{13}$ & 2 \\
\hline & & \\
\hline Oración aseverativa & Predicado & Nombre propio \\
\hline 'Juan es músico' & ' $\mathrm{x}$ es músico' & Juan \\
\hline
\end{tabular}

Ahora bien, si lo que interesa es la validez de los argumentos a partir de oraciones aseverativas verdaderas o falsas, es necesario según Frege contar con una teoría general sobre el modo en que se determina la verdad o falsedad de una oración aseverativa a partir de sus elementos constituyentes, nombres propios y predicados. Esto es, contar con una semántica o teoría del significado (Bedeutung) para las expresiones sintácticas, esto es:

\begin{tabular}{|c|c|c|}
\hline \multicolumn{2}{|c|}{ Semántica } \\
\hline Oración aseverativa & Predicado & Nombre propio \\
\hline$\downarrow$ & $\downarrow$ & $\downarrow$ \\
Su significado es & Su significado es & Su significado es \\
\hline Lo verdadero o lo falso & Un concepto & Un objeto \\
\hline
\end{tabular}

A estos tres elementos se los llama también como "propiedades semánticas" de la oración y de sus partes. Si a los elementos del nivel sintáctico le sumamos los cuantificadores, también invención fregeana, llegamos al cuadro de oposición de más arriba.

Ahora bien, lo que interesa para nuestro trabajo es lo siguiente: por el principio de composicionalidad del significado (fundamental para Frege), una oración aseverativa

12 “Während in der vorhin angedeuteten Betrachtungsweise 'Wasserstoffgas' das Argument, 'leichter als Kohlensäuregas zu sein' die Function war, können wir den selben begrifflichen Inhalt auch in der Weise auffassen, dass 'Kohlensäuregas' Argument, 'schwerer als Wasserstoffgas zu sein' Function wird".

13 En sentido estricto esta expresión no es una función sino hasta que tome un segundo elemento, por ejemplo, el 5. Obtenemos lo siguiente: ' $\mathrm{x}+3=\mathrm{y}$ '. De igual modo, y he aquí un gran descubrimiento de Frege, a partir de 'Juan ama a María' obtengo la expresión ' $\mathrm{x}$ ama a y'. En principio esto último es una relación y si cumpliera biyección (suryección e inyección) sería una función. 
es verdadera o falsa si y solo si sus componentes tienen significado. Es decir, (i) al predicado le corresponde un concepto y (ii) al nombre propio un objeto. Veamos a continuación las consecuencias de lo primero.

\section{Predicados y compromiso ontológico: vacuidad y subalternación}

La exigencia semántica de que a los predicados les corresponda un concepto no contiene ningún compromiso ontológico (a menos que uno defienda que la realidad es la de los conceptos [Platón]). Es decir, los predicados pueden ser vacíos y esto no pone en cuestión sus propiedades semánticas. Ocurre lo contrario con los nombres propios, pero lo veremos en la sección final. ¿Qué es -semánticamente hablando- un predicado vacío? Pues,un predicado del cual todo lo que se predique es falso. Por ejemplo, el predicado " $x$ es el más grande de los números naturales", incluso si reemplazo todos los números que humanamente me sea posible. Ahora bien, si un predicado puede ser vacío, la relación de subalternación desaparece. Esto se puede probar de modo sencillo buscando un predicado $P x$ vacío que hará verdadero el condicional de la universal y falsa la conjunción del particular.

$$
\begin{array}{|l|}
\forall x(P x \rightarrow Q x) \rightarrow \exists x\left(P x^{\wedge} Q x\right) \\
\text { F } \\
\text { V F } \\
\text { V F }
\end{array}
$$

Una curiosidad es que esta versión de la subalternación que damos a continuación (si se nos permite llamarla así), sí es válida: $\forall x P x \rightarrow \exists x P x$. Aquí una prueba dialógica sencilla:

\begin{tabular}{|c|c|c|c|c|c|}
\hline & $\mathrm{O}$ & & & $\mathrm{P}$ & \\
\hline & & & & $\forall x P x \rightarrow \exists x P x$ & 0 \\
\hline 1 & $\forall x P \mathrm{x}$ & 0 & & $\exists x P x$ & 2 \\
\hline 3 & $? \exists$ & 2 & 1 & $P k_{1} \odot$ & 6 \\
\hline 5 & $P k_{1}$ & & 1 & $? k_{1}$ & 4 \\
\hline
\end{tabular}

\section{El cuantificador existencial y su arraigo telúrico}

Este tridente que nos da la espalda no es otra cosa que la letra "E" de "Exist" pero al revés, y tiene poderes ontológicamente discriminatorios. Tanto en su versión A como en la B (Gamut 2002: 94-100), este predicado de segundo orden, el único [permitido] dentro de la lógica de primer orden, nos "exige" que el lenguaje de la lógica se ocupe 
de cosas que existan en el mundo. Ahora bien, qué cosas existen en el mundo no es problema de la lógica, dicen los lógicos clásicos. Si esto es así, dirán los detractores de la lógica clásica, podemos entonces usar la lógica para determinar lo V o lo F dentro de una historia de ficción. Entonces, los lógicos clásicos sentencian: ningún problema señores detractores, úsenla (como si se tratara de seres "existentes", que no lo son) pero todo lo que afirmen será falso. ¿Cuál es el problema con este enfoque? Pues, que allí donde queramos establecer la diferencia, no será posible en términos de lógica clásica. Porque la existencia se determina con un predicado de segundo orden que nos habla de la existencia o no existencia de individuos que corresponden a un concepto. Si una ausencia se impone en lógica clásica es la de un predicado de primer orden de existencia. En definitiva, a modo de ejemplo, no es posible decir que es verdad que 'Harry Potter usa lentes como Gandhi', pues el segundo está dentro del alcance del cuantificador, el primero no.

9. Nombres propios y compromiso ontológico: la esquizofrenia de la lógica clásica y la búsqueda de la neutralidad

Como se habrá percatado el lector, mientras que la propiedad semántica fundamental de los predicados es su correspondencia con conceptos (que parece que solo Platón sabe dónde están), con los nombres propios la lógica establece una exigencia cuasi prosaica y pedestre. En efecto, los objetos que son la propiedad semántica que deben reunir los nombres propios para la determinación de la verdad o falsedad de las oraciones aseverativas deben hallarse como existentes en el mundo que habitamos. Lo repetimos: mientras que para los predicados no hay problema con la existencia o no existencia de cosas en el mundo a las cuales se aplique (o corresponda), con los nombres propios no podemos imaginar una exigencia mayor: que exista el portador de cada nombre propio. O para hacerlo ontológicamente más vertiginoso, que exista un portador de cada término singular $\mathrm{k}_{\mathrm{n}}$, para $\mathrm{n}$ entre $1 \mathrm{y}$ el infinito (numerable). ¿Habrá suficientes números para cada cosa del universo? No entraremos en este tema aquí, solo recordamos que Dedekind $(1872 ; 1888)$ y Cantor $(1874 ; 1879)$ tuvieron mucho que decir al respecto.

Karel Lambert califica este desnivel o desarmonía de los compromisos ontológicos de predicados y nombres propios como la esquizofrenia de la lógica clásica (Lambert 2003: 142). Y su propuesta no es nivelar hacia los nombres propios sino hacia lo que caracteriza a los predicados. Es decir, propone alcanzar una semántica que se ponga a resguardo de compromisos mayores con el mundo. De hecho, se propone que los términos utilizados en lógica no tengan ningún compromiso ontológico. Todos los caminos que van en esta dirección nos alejan de la lógica clásica.

Es decir, el objetivo de Lambert, y también de otros autores como es el caso de Bencivenga (2002), es la neutralidad de la lógica clásica frente a las determinaciones de lo verdadero y lo falso (su semántica). ¿Si ha de llamarse formal la lógica que hacemos y desarrollamos, por qué tanto apego a lo existente? ¿No es curioso? Es como si para saber si es verdad o no que $2+3=5$, fuera necesario reunir objetos existentes en el mundo que "verifiquen" tal adición. No hay que olvidar que, si en la frutera tenemos 
solo dos manzanas y tres naranjas, la "verificación" no será posible. Deberemos ir al mercado de la esquina y procurar reunir o bien cinco naranjas o bien cinco manzanas.

¿Y cómo lograr tal neutralidad? De diferentes modos, pero el más conocido es el camino que toman las llamadas lógicas libres (Free Logics). Este nombre es una abreviación para "lógicas libres de compromisos ontológicos" y según Lambert (1997: 35) se definen así: "logic free of existence assumptions with respect to its general and singular terms, but whose quantifiers are interpreted exactly as in classical first order predicate logic".

Esta definición nos recuerda que la restricción de la lógica clásica para el uso de los nombres propios (o términos singulares) corresponde al alcance de los cuantificadores. Es decir, los portadores de los nombres propios son aquellas mismas cosas que están dentro del alcance de los cuantificadores, esto es, la totalidad de lo existente (como lo entiende la lógica clásica). La lógica libre, contrariamente,se permite una extensión del uso de los nombres propios, más allá de los cuantificadores clásicos. ¿Cómo la realiza? Veámoslo en lo que sigue.

El primer objetivo de este tipo de lógicas es suprimir la restricción que ata los nombres propios a los objetos del mundo que habitamos. Esta supresión es tan resistida como temida por los lógicos clásicos, no tanto por los alambicados cambios sintácticos que requiere sino por sus consecuencias semánticas. En efecto, no debemos olvidar cuáles fueron las motivaciones originales de Frege. La consecuencia semántica más buscada por él -por el contrario- fue la de mantener a raya a las ficciones literarias. Si hay que diferenciar entre el lenguaje científico y su compromiso con la verdad y la falsedad al hablar de las cosas del mundo (la física, la biología, etc.), debe mantenerse cerrada la posibilidad de poder predicar con verdad o falsedad sobre cosas que no existen. ¿Y cómo lo logra Frege? Sencillamente prohibiendo el uso de nombres vacíos (nombres que no refieren a nada) o prohibición de usar nombres propios para lo que no existe. Aquello que se predica usando nombres vacíos (al estilo "Ulises era hombre"), es decir, sobre lo que no puede haber ciencia o conocimiento según esta tradición, no es otra cosa que literatura y poesía. Porque lo verdadero y lo falso -para Frege y los restantes lógicos clásicos- pertenecen al reino de la ciencia y la búsqueda de conocimiento y no de la ficción. Denodados esfuerzos hizo Frege por mantener lejos a las ficciones aunque contrariamente por culpa de ellas -según su propia confesión-se derrumbó el logicismo por el cual trabajó toda su vida:

Una propiedad funesta del lenguaje para la confiabilidad del pensar es su tendencia a formar nombres propios sin objeto alguno que les corresponda [...] Un ejemplo particularmente curioso de esto es la formación de un nombre propio según el modelo "la extensión del concepto [F]", p. Ej., "la extensión del concepto estrella fija". Esta expresión parece designar un objeto debido al artículo definido; pero no hay ningún objeto que lingüísticamente pudiera designarse de este modo. De aquí se han originado las paradojas de la teoría de conjuntos, paradojas que la han aniquilado. Yo mismo estoy sometido a esta ilusión en el intento de fundamentar lógicamente los números, mientras quería considerarlos como conjuntos" (Frege 1983: 288s. Itálicas nuestras. Traducción tomada de Stepanians 2007: 96). 
Ahora bien, volviendo a Lambert, ¿cómo se suprime la restricción que ata los nombres propios a los objetos del mundo que habitamos? Invalidando Especificación ( $\forall x A$ $\vdash A(t / x))$ y Particularización o generalización existencial $(A(t / x) \vdash \exists x A)$, teoremas fundamentales del sistema axiomático primordial de la lógica clásica. En la primera versión de 1879 de la Conceptografía de Frege aparece Especificación en la primera lista de axiomas fundamentales.

\section{¿Qué nos dicen o exigen Especificación y Particularización?}

La Especificación nos exige que exista al menos un individuo que verifique o posea la propiedad que he predicado universalmente. Un ejemplo: Si es verdad que todos los perros son mamíferos, entonces debe ser verdad que existe al menos un perro (con nombre propio) que es mamífero.

La Particularización, por su parte, exige que,si predico con verdad algo de un individuo o cosa, debe existir al menos un individuo o cosa que cumpla con esa propiedad. Otro modo de entenderlo es siguiendo el llamado principio de predicación (Reicher 2014):

$$
F b \rightarrow \exists x(x=b)
$$

Esto se lee de dos modos: si $b$ es $F$, entonces existe algo que es idéntico a $b$; si $b$ es $F$, entonces $b$ existe.

En definitiva, ninguna de éstas es válida considerando los personajes de una obra de ficción. Desembarazándonos de ellas será posible pensar en condiciones de verdad para oraciones que, para ser verdaderas o falsas, no deban tener un férreo compromiso con la existencia de las cosas. Desde luego se pueden elaborar distintas semánticas sin este férreo compromiso, dependiendo de la perspectiva que se elija ${ }^{14}$.

A continuación, presentaremos una alternativa semántica que no posee restricciones ontológicas como la lógica clásica.

\section{El rol de la interacción en lógica: la elaboración de una semántica lúdica}

¿Es posible otra semántica? Es decir, ¿otra forma de entender el significado? Es bien conocida la idea de Wittgenstein de que, en un gran número de casos, comprender la significación de una expresión significa conocer el uso que se hace de esta expresión en el contexto de una interacción lingüística, que a su vez es comprendido como un juego (Wittgenstein 1953). Sabemos que Wittgenstein nunca dio una definición precisa de

14 En particular, para la semántica de Lambert, cf. Lambert, $(1960 ; 1983 ; 1991)$. En la misma dirección, tenemos los desarrollos de los meinongianos, cf. (Priest 2005). 
juegos de lenguaje y por una buena razón, puesto que él defiende la idea de que esos juegos a menudo están desprovistos de reglas y, por tanto, sin una forma determinada.

En efecto, en el seno de la lógica matemática del siglo XX, surgió un conjunto de técnicas, conceptos y resultados que constituyeron una suerte de paradigma en el cual la idea de inferencia lógica es un caso particular de la interacción entre los participantes de un diálogo crítico. El vocabulario filosófico presenta a menudo la siguiente ambigüedad (cf. Per Martin-Löf 1996): un mismo término designa a la vez una acción y el contenido o resultado de dicha acción. Es el caso, entre otros, de "razonamiento" y "proposición" en la perspectiva de Frege. Johan van Benthem (1994: 109) señala que esta ambivalencia, que oscila entre un polo "estático" (el contenido) y otro "dinámico" (la acción), confirma las diferentes representaciones de lo que debe ser la tarea propia de la lógica.

Para la tradición de la lógica matemática que culmina en la perspectiva de Frege, la lógica es el estudio de una estructura compuesta de proposiciones y de relaciones entre esas proposiciones. Entre estas últimas, la de consecuencia lógica es la más importante. Pero a partir de los años treinta, una nueva corriente piensa que la teoría de la significación y de los contenidos de pensamiento (tradición estática), debe ir acompañada de la teoría del acto de pensar o de significar (punto de vista dinámico). Podemos considerar el intuicionismo de L.E.J. Brouwer $(1907$; 1908) como el punto de partida de esta tradición.

La estructura proposicional que es objeto de la tradición estática o clásica se define semánticamente como una estructura booleana, donde las proposiciones son consideradas como valores de verdad y las constantes lógicas como operadores sobre esos valores. Sintácticamente, como un álgebra de signos puros sobre los cuales operamos vía reglas de cálculo. La existencia de tales estructuras es considerada como un hecho matemático, y su adecuación para dar las normas del razonamiento como una evidencia. Por ello, en esta perspectiva, con palabras de van Benthem:

[...] el énfasis reside en el hecho de "que" o de "si" ciertas oraciones son verdaderas respecto de una situación, pero no tanto de "cómo" llegaron ellas a ser consideradas como verdaderas (van Benthem 1994: 109. Nuestra traducción).

\section{Crítica a la doble negación y el tercero excluido: el rol del intuicionismo}

La cuestión de hacer del "cómo" el interrogante principal de la lógica, posee consecuencias importantes, tanto filosóficas como técnicas. Es aquí, justamente, donde la lógica intuicionista entra en juego en tanto que es ella la primera tentativa de desarrollar estas consecuencias. En efecto, hay al menos dos principios que son considerados como válidos para la lógica clásica pero que se presentan como problemáticos para quienes pretenden considerar el modo de aprehensión de la verdad de un enunciado por un sujeto de conocimiento: el primero es la doble negación, el segundo es el tercero excluido. 
El primero es el núcleo de un modo de inferencia crucial en matemáticas: el razonamiento por el absurdo. Deducir $A$ a partir de su doble negación, según los intuicionistas, genera problemas que conciernen directamente al cuantificador existencial: podemos mostrar por el absurdo la existencia de entidades matemáticas sin necesidad de exhibirlas o de construirlas, lo cual pone en cuestión la significación del cuantificador. Parece más razonable exigir, si lo que nos interesa es el modo de aprehensión de la verdad de un enunciado, que la condición de reconocimiento de la verdad de un existencial sea la capacidad de determinar un valor particular para la variable cuantificada, de tal modo que el enunciado de la fórmula correctamente instanciada sea verdadero.

Respecto del tercero excluido, el argumento que demuestra su validez esconde una sutileza inaceptable para los intuicionistas: la demostración de la disyunción principal es realizada sin que ninguno de los dos miembros de la disyunción sea probado. Lo razonable, argumentan, es que la demostración se lleve a cabo como una demostración por un miembro o por el otro (tal y como es definido el comportamiento de una disyunción en teoría de la demostración). En otras palabras: la demostración del tercero excluido se apoya en el razonamiento por el absurdo o en una estructura más compleja en la cual no se tiene en cuenta la demostración de los componentes de la disyunción. Como bien lo remarca Dummett (1977), si no queremos considerar una teoría de la verdad de modo independiente de una teoría del modo de reconocimiento de esa verdad, el tercero excluido resulta inaceptable puesto que nos fuerza a considerar en una demostración la existencia de demostración que no poseemos.

Por todo esto, el lógico que decide tener en cuenta el reconocimiento de la verdad, bajo la forma de una teoría de la construcción de demostraciones o de una epistemología de los medios de verificación, es conducido sin retraso a modificar su concepción de las leyes de la lógica, lo que da lugar a las lógicas no clásicas.

\section{Ausencia y necesidad de una semántica para el intuicionismo}

Sin embargo, el desarrollo de la lógica intuicionista encuentra una dificultad mayor de orden semántico. Para la estructura proposicional que es objeto de estudio de la lógica clásica, se proporciona una noción de semántica desarrollada a partir de los trabajos de Alfred Tarski (1983) y conocida como teoría de modelos. Esta teoría se hace cargo de la noción de verdad vía la noción de referencia: a partir de una función de interpretación de términos individuales y de predicados, es posible hacer explícito el valor de verdad de un enunciado relativo a la estructura. Pero, aquí lo problemático, la definición tarskiana de modelos presupone la validez del tercer excluido y, por tanto, la lógica intuicionista emerge como un cálculo puro sin que se le pueda asociar a una semántica entendida en el sentido de una teoría de la referencia (una semántica referencialista). Posibles soluciones a esta situación provienen de dos caminos hasta cierto punto emparentados. 
En efecto, en general tenemos dos tradiciones que afirman implementar la noción de juegos de lenguaje en lógica. Por un lado, la lógica de Lorenzen y Lorenz $(1955,1978)$ que nació directamente de la intención de dar a la lógica intuicionista una semántica propia. Por otro, la semántica de juegos de Hintikka (la GTS=semántica de juegos), con un origen independiente y que por razones de espacio no desarrollaremos aquí.

\section{Lógica dialógica}

Lo que está en cuestión aquí es saber hasta qué punto es posible que la noción procedural de demostración, que normalmente está ausente de las prácticas lingüísticas corrientes, pueda otorgar una semántica a los enunciados. Y es, en efecto, en la noción de diálogo donde Lorenzen y Lorenz (1978) encuentran el concepto que permite explicar el significado de las constantes lógicas (o partículas). La noción de diálogo guarda intactas las intuiciones lingüísticas corrientes remarcando la importancia de la dimensión procedural y epistémica de la noción de demostración.

¿Qué son los diálogos? Los diálogos son juegos de lenguaje matemáticamente definidos para que establezcan la interfaz entre la actividad lingüística concreta y la noción formal de demostración. Dos interlocutores (proponente y oponente) intercambian movimientos que son concretamente actos lingüísticos. El proponente enuncia una tesis, la tesis del diálogo, y se compromete a defenderla respondiendo a todas las críticas del oponente. Las críticas permitidas son definidas en términos de la estructura de los enunciados afirmados en el diálogo. Por ejemplo, si un jugador afirma la conjunción A y B, al mismo tiempo concede al adversario la posibilidad de elegir uno de los dos y de exigirle que lo afirme. La noción misma de afirmar se encuentra definida por el contexto de la interacción crítica: afirmar significa comprometerse a proporcionar una justificación a un interlocutor crítico. Pero en diálogos no hay una teoría general de la justificación sino solo en la medida en que se trate de enunciados lógicamente complejos que encuentran su justificación a partir de enunciados simples. A su vez, los enunciados simples se justifican en acción recíproca con el interlocutor crítico. Esto es, según exhorta la regla, el proponente podrá considerar justificado un enunciado elemental, si y solamente si el oponente ha concedido esa justificación. Esta regla confirma la formalidad de los diálogos: el proponente gana sin presuponer justificaciones por ningún enunciado particular. También gana la dialógica su condición de formal en tanto no dibuja ninguna descripción ontológica del mundo.

Las reglas que definen el enfoque dialógico son de dos tipos: reglas de partículas y reglas estructurales. Las reglas de partículas describen de forma abstracta el modo en que una fórmula puede ser atacada y defendida en función de su conectiva principal. Ellas corresponden al significado de las conectivas en dialógica y son las que caracterizan este paradigma: 


\section{Cuadro 1}

\begin{tabular}{|c|c|c|c|}
\hline Partículas & Aserción & Ataque & Defensa \\
\hline \multirow[t]{2}{*}{$\wedge$} & \multirow[t]{2}{*}{$\mathrm{X}-!-\mathrm{A} \wedge \mathrm{B}$} & $Y-?-\wedge_{1}$ & $\mathrm{X}-!-\mathrm{A}$ \\
\hline & & $Y-?-\wedge_{2}$ & X-!-B \\
\hline V & $X-!-A \vee B$ & Y-?-V & X-!-A o X-!-B \\
\hline$\rightarrow$ & $\mathrm{X}-!-\mathrm{A} \rightarrow \mathrm{B}$ & Y-!-A & $\mathrm{X}-!-\mathrm{B}$ \\
\hline$\neg$ & $\mathrm{X}-!-\neg \mathrm{A}$ & Y-!-A & No hay \\
\hline$\forall$ & $X-!-\forall x \varphi$ & $\begin{array}{c}\mathrm{Y}-?-\mathrm{x} / \mathrm{k}_{\mathrm{i}} \\
\mathrm{Y} \text { tiene la elección }\end{array}$ & $X-!-\varphi\left[x / k_{i}\right]$ \\
\hline$\exists$ & $X-!-\exists x \varphi$ & $\mathrm{Y}-?-\exists \mathrm{x}$ & $\begin{array}{c}\mathrm{X}-!-\varphi\left[\mathrm{x} / \mathrm{k}_{\mathrm{i}}\right] \\
\mathrm{X} \text { tiene la elección }\end{array}$ \\
\hline
\end{tabular}

Las reglas estructurales, por el contrario, especifican la organización general del juego (para más detalle, ver el Apéndice).

Ciertamente, lo que muestra en definitiva el tratamiento dialógico del razonamiento son las condiciones formales de un acuerdo entre dos jugadores que han fijado reglas para un debate. Cabe recordar aquí la actitud argumentativa de Sócrates. En efecto, un diálogo quedará lógicamente decidido si aquel que propone la tesis funda sus ventajas argumentativas no en pruebas externas al debate (tan numerosas como son), sino en la evidencia de su mismo oponente:

En cuanto a mí, no creo haber formulado ninguna conclusión que valga la pena acerca del asunto de nuestra disputa, a menos que no te reduzca a que te presentes tú mismo a rendir testimonio de la verdad de lo que digo; y tú creo que nada podrás alegar contra mí a menos que yo, que estoy solo, declare en tu favor y que no asignes importancia al testimonio de los otros. He aquí, pues, dos maneras de refutar: la una la que tú y otros creéis verdadera, y la otra la que yo, por mi parte, juzgo verdadera (Platón 1987: 472b-c, pp. 61-62).

Esta forma de refutación se encuentra, mutatis mutandis, en la lógica dialógica: la tesis no es considerada como formalmente justificada sino a condición de que esa justificación sea producida en función de la significación de constantes lógicas y de las justificaciones elementales concedidas por el adversario. En definitiva, lo que tenemos con la dialógica es una semántica para la lógica intuicionista, esto es, una teoría del significado que no es una teoría de la referencia.

\section{A modo de conclusión: lógica dialógica y ficciones}

Para finalizar, quisiéramos delinear brevemente como proyecto la perspectiva dialógica del análisis del discurso ficcional que se sigue de estas consideraciones. 
A menudo encontramos en la literatura especializada objeciones contra el pretendido poder explicativo de la lógica y de los lenguajes formales en relación con el discurso ficcional. El objetivo de tales críticas es generalmente el lugar central que ocupa para la lógica la noción de referencia en el análisis de la ficción, mientras que lo más idóneo se encontraría en un abordaje más pragmático. A esta objeción respondemos afirmando que, si elaboramos un contexto de análisis adecuado, es posible un tratamiento propiamente pragmático de la ficción en lógica y sin las restricciones que impone la noción de referencia. Y la lógica dialógica, que considera los argumentos como un encadenamiento de preguntas y respuestas, se impone como un contexto de análisis ideal para este abordaje más pragmático.

El aspecto más determinante de la contribución de la perspectiva dialógica al análisis de la ficción es que no entiende la existencia como un predicado o propiedad o presupuesto, sino en función de una acción: la acción de elegir un individuo que, al mismo tiempo, constructivamente se constituye como tal (cf. Regla del cuantificador existencial en el Cuadro 1). Respecto de los cuantificadores, la contribución de la dialógica lleva a no desfavorecer una relación crucial para su significación y que queda oscurecida en las otras perspectivas: la relación entre la elección de una constante de sustitución y la aserción que resulta de esta acción.

En este sentido creemos en la riqueza de la perspectiva de la lógica dialógica que comprende la existencia a través del concepto interaccional de elección, cuya su semántica se basa en el concepto de utilización y puede denominarse como semántica pragmática. El contraste que establece esta comprensión de la existencia con las otras perspectivas permite pensar en un punto de vista estático que entiende la existencia como un predicado frente a un punto de vista dinámico que se centra en la noción interaccional de elección (Cf. Rahman 2001, 2004 y 2010; Redmond 2010).

Una de las más fructíferas consecuencias de los dispositivos puestos en funcionamiento en esta perspectiva es que el estatus ontológico de las constantes y el compromiso ontológico de los cuantificadores pueden variar a lo largo de una prueba. Asimismo, este esquema de análisis puede completarse considerando las ficciones, nuevamente de modo dinámico, en relación con un acto creativo. Creemos posible esto último implementando la noción fenomenológica de dependencia ontológica (Fontaine, Redmond y Rahman 2009) instrumentada en un sistema dialógico libre para un lenguaje de primer orden. En un trabajo futuro daremos cuenta de modo detallado de la integralidad de esta propuesta. 


\section{Apéndice \\ Una presentación técnica condensada de lógica dialógica estándar ${ }^{15}$}

Sea L un lenguaje de primer orden construido en base a conectivas proposicionales, cuantificadores, un conjunto numerable de variables individuales, un conjunto numerable de constantes individualesy un conjunto numerable de símbolos de predicado (cada uno con una aridad fija).

Extendemos el lenguaje L con dos etiquetas $\mathrm{O}$ y $\mathrm{P}$ que corresponden a los participantes del diálogo, y el signo de interrogación"?". Cuando la identidad del jugador no importa, utilizamos variables $\mathrm{X}$ o $\mathrm{Y}(\operatorname{con} \mathrm{X} \neq \mathrm{Y})$. Un movimiento es una expresión de la forma $\mathrm{X}-e$, donde $e$ es o bien una proposición $A$ de $\mathrm{L}$ o una de las expresiones siguientes: ? ${ }_{\wedge \mathrm{i}(\mathrm{i}=\mathrm{L} \mathrm{o} i=\mathrm{R}),} ?_{\vee}, ?_{[A(\mathrm{a} / \mathrm{x})]}, ?_{[A(a 1 / x), \ldots, A(a \mathrm{an} / x)]}$

Hay dos tipos distintos de reglas llamadas reglas de partículas (que proveen el significado local) y reglas estructurales (que proveen el significado global). Comenzamos conlas reglas de partículas:

\begin{tabular}{|c|c|c|c|c|}
\hline Movimiento previo & $\mathrm{X}-A \wedge B$ & $\mathrm{X}-A \vee B$ & $\mathrm{X}-A \rightarrow B$ & $\mathrm{X}-\neg A$ \\
\hline Ataque & $\begin{array}{l}\mathrm{Y}-?_{\wedge \mathrm{L}} \mathrm{O} \\
\mathrm{Y}-?_{\wedge 2}\end{array}$ & $\mathrm{Y}-?_{\vee}$ & Y- $A$ & $\mathrm{Y}-A$ \\
\hline Defensa & $\begin{array}{c}\mathrm{X}-A \\
\text { resp. X-B }\end{array}$ & $\begin{array}{c}\mathrm{X}-A \\
\text { o X- } B\end{array}$ & $\mathrm{X}-B$ & -- \\
\hline
\end{tabular}

\begin{tabular}{|c|c|c|}
\hline Movimiento previo & $\mathrm{X}-\forall x A$ & $\mathrm{X}-\exists x A$ \\
\hline Ataque & $\mathrm{Y}-?_{[A(a / x)]}$ & $\mathrm{Y}-?_{[A(a 1 / x), \ldots, A(a \mathrm{an} / x)]}$ \\
\hline Defensa & $\mathrm{X}-A(a / x)$ & $\mathrm{X}-A\left(a_{\mathrm{i}} / x\right)$ \\
& & con $1 \leq \mathrm{i} \leq \mathrm{n}$ \\
\hline
\end{tabular}

En esta tabla, una expresión de tipo $a_{i}$ es una constante individual y $A\left(a_{i} / x\right)$ expresa la proposición obtenida mediante la sustitución de cada ocurrencia de $x$ en $A$ por $a_{i}$. Cuando un movimiento consiste en una pregunta de la forma ' ${ }_{[A 1, \ldots, A \mathrm{n}]}$ ' o de la forma '? ${ }_{\vee}$, , el otro jugador elige una proposición entre $A_{1}, \ldots, A_{\mathrm{n}}$ y la juega. Así, podemos -en términos de qué jugador tiene una opción- distinguir entre la conjunción y disyunción, por una parte, y la cuantificación universal y la existencial, por otra. En los casos de la conjunción y la cuantificación universal, el retador (o atacante) elige, mediante un ataque de la forma

15 La siguiente presentación de lógica dialógica estándar ha sido resumida de Clerbout (2013, 2014a) y ligeramente adaptada a la notación utilizada en el presente artículo. 
'? ${ }_{\wedge \mathrm{i}}$ ' (para la conjunción) o de la forma '? ${ }_{[A(a / x)]}$ ' (para el universal) la proposición por la cual preguntar. Por el contrario, en los casos de disyunción y cuantificación existencial, el defensor es el único que puede elegir entre varias proposiciones. Obsérvese que no hay defensa en el caso de la regla de partículas para la negación.

Las reglas de partículas proporcionan una descripción abstracta de cómo se procede en el diálogo a nivel local: especifican el modo en el que una proposición puede atacarse y defenderse de acuerdo con su constante lógica principal. Decimos que tales reglas gobiernan el nivel local del significado. En rigor, las expresiones que aparecen en la tabla más arriba no son movimientos reales porque tienen proposiciones esquemáticas y los jugadores no están especificados. Además, estas reglas son indiferentes al rol de la proposición en las diversas variedades de diálogos en los que pueda intervenir: por ejemplo, las reglas locales de las constantes lógicas no varían si los diálogos son clásicos o intuicionistas. Por este motivo decimos que la descripción dada por las reglas de partículas es en cierto modo abstracta.

Las expresiones "ataque" y "defensa" son convenientes para prescribir ciertas interacciones entre movimientos. Tales interacciones pueden ser definidas con precisión de la forma siguiente. Sea $\Sigma$ una secuencia de movimientos. La función $\mathrm{p}_{\Sigma}$ asigna una posición para cada movimiento en $\Sigma$, comenzando con 0 . La función $\mathrm{F}_{\Sigma}$ asigna un par $[\mathrm{m}$, Z] para ciertos movimientos $\mathrm{N}$ en $\Sigma$, donde $m$ denota una posición menor que $\mathrm{p}_{\Sigma}(\mathrm{N}) \mathrm{y} Z$ es o bien $\mathcal{A}$ (un ataque)o bien $\mathcal{D}$ (una defensa). Es decir, la función $\mathrm{F}_{\Sigma}$ permite seguir la "historia" de las interacciones ataque-defensa que originaron un movimiento dado. Un diálogo (o partida) es una secuencia legal de movimientos, es decir, una secuencia de movimientos que observa las reglas del juego. La segunda clase de reglas que hemos mencionado, las reglas estructurales, dan las condiciones exactas en las que una oración dada genera un juego dialógico. Un juego dialógico para $A$, escrito $\mathcal{D}(A)$, es el conjunto de todas las partidas con $A$ como la tesis (ver la regla de inicio más abajo). Las reglas estructurales son las siguientes:

SRO (Regla de inicio). Sea $A$ una proposición compleja ${ }^{16}$ de L. Para cada $\pi \in \mathcal{D}(A)$ tenemos:

$$
\begin{aligned}
& -p_{\pi}(\mathrm{P}-A)=0, \\
& -p_{\pi}(\mathrm{O}-\mathrm{n}:=i)=1, \\
& -p_{\pi}(\mathrm{P}-\mathrm{m}:=j)=2
\end{aligned}
$$

En otras palabras, cualquier partida $\pi$ en $\mathcal{D}(A)$ comienza con P- $A$. Llamamos $A$ a la tesis de la partida y del juego dialógico correspondiente. Después de eso, el Oponente y el Proponente eligen sucesivamente un número entero positivo llamado rango de repetición. El papel de este entero es asegurar que cada partida termine después de

16 Si la tesis es una proposición elemental, hay que implementar una pequeña modificación de la regla formal SR2, como detallamos más abajo. 
un número finito de movimientos, de una manera especificada por la siguiente regla estructural.

\section{SR1 (Regla clásica)}

- Sea $\pi \in \mathcal{D}(A)$. Para cada $M$ en $\pi$ donde $p_{\pi}(M)>2$ tenemos $F_{\pi}(M)=\left[m^{\prime}, Z\right]$ donde $m^{\prime}<p_{\pi}(M)$ y $Z \in\{\mathcal{A}, \mathcal{D}\}$

- Sea $r$ el rango de repetición del jugador $\mathrm{X}$ y $\pi \in \mathcal{D}(A)$ tal que

- el último miembro de $\pi$ es un movimiento de $\mathrm{Y}$,

- $M_{0}$ es un movimiento de $\mathrm{Y}$ de posición $m_{0}$ en $\pi$,

$-M_{1}, \ldots, M_{\mathrm{n}}$ son los movimientos de X en $\pi$ tal que $F_{\pi}\left(M_{1}\right)=\ldots=F_{\pi}\left(M_{\mathrm{n}}\right)=\left[m_{0}, Z\right]$.

Considérese la secuencia ${ }^{17} \pi^{\prime}=\pi_{*} N$ donde $N$ es un movimiento de $\mathrm{X}$ tal que $\mathrm{F}_{\pi^{\prime}}(N)=\left[m_{0}\right.$, $Z$ ]. Tenemos $\pi^{\prime} \in \mathcal{D}(A)$ solo si $\mathrm{n}<r$.

La primera parte de la regla establece que cada movimiento, después de la elección de los rangos de repetición, es o bien un ataque o una defensa. La segunda parte se asegura la finitud de las partidas mediante el establecimiento de un rango de repetición del jugador como el número máximo de veces que puede desafiar o defenderse de un movimiento determinado de otro jugador.

SR2 (Regla formal). Sea $B$ una proposición elemental, $N$ el movimiento P- $B$ y $M$ el movimiento O- $B$. Una secuencia $\pi$ de movimientos es una partida solo si se cumple: si $N \epsilon \pi$ entonces $M \epsilon \pi$ y $p_{\pi}(M)<p_{\pi}(N)$.

Es decir, si el Proponente afirmó una proposición elemental, entonces O la afirmó ya antes. En el caso de juegos en los que se permite que la tesis sea una proposición elemental, hay que reformular la regla formal de la siguiente manera:

SR2* (Regla formal modificada). O puede atacar una proposición atómica si y solo si él mismo no la afirmó aún. Solo el oponente puede atacar proposiciones atómicas. El proponente se defiende de un ataque a una proposición atómica, mostrando que en el ulterior desarrollo del juego el oponente será forzado a conceder la proposición atómica atacada, digamos en el movimiento $n$. En cuanto $\mathrm{O}$ jugó $\mathrm{n}$, entonces $\mathrm{P}$, se defiende del ataque, respondiendo sic (n) (léase: acabas de conceder en $n$ la proposición atómica buscada).

Decimos que una partida es terminal cuando no puede ampliarse en sucesivos movimientos legales. Decimos que es X-terminal cuando el último movimiento en la partida es un movimiento del jugadorX. 
SR3 (Regla de ganancia). El jugador X gana la partida $\pi$ solo si es terminal X.

Considérese por ejemplo las siguiente secuencia de movimientos: $\mathrm{P}-Q \mathrm{a} \rightarrow Q a, \mathrm{O}-\mathrm{n}:=1$, $\mathrm{P}-\mathrm{m}:=12, \mathrm{O}-Q a, \mathrm{P}-Q a$, que pueden ser escritas del siguiente modo:

\begin{tabular}{|c|c|c|c|c|c|}
\hline & $\mathrm{O}$ & & & $\mathrm{P}$ & \\
\hline & & & & $Q \mathrm{a} \rightarrow Q a$ & 0 \\
\hline 1 & $\mathrm{n}:=1$ & & & $\mathrm{~m}:=12$ & 2 \\
\hline 3 & $Q a$ & $(0)$ & & $\mathrm{Qa}$ & 4 \\
\hline
\end{tabular}

Los números de las columnas externas son las posiciones de los movimientos en la partida. Cuando un movimiento es un ataque, la posición del movimiento desafiado se indica en las columnas internas, como ocurre con movimiento 3 en este ejemplo. Nótese que este tipo de tablas llevan la información facilitada por las funciones p y F, además de representar la partida en sí.

Sin embargo, cuando queremos considerar varias partidas juntas -por ejemplo, en la construcción de una estrategia- dichas tablas no proporcionan el medio de representación más adecuado. De hecho, cuando queremos representar la construcción de una estrategia usamos lo que se conoce como la forma extensiva. La forma extensiva del diálogo $\mathcal{D}(A)$ es simplemente la representación del árbol del mismo, también a menudo llamado árbollúdico. Más precisamente, la forma extensiva $\mathrm{E} A$ de $\mathcal{D}(A)$ es el árbol $(T, l, S)$ tal que:

i) Cada nodo $t$ en $T$ está etiquetado con el movimiento que ocurre en $\mathcal{D}(A)$.

ii) $l: T \rightarrow \mathrm{N}$

iii) $S \subseteq T^{2}$ donde:

- Hay un único $t_{0}$ (la raíz) en $T$ tal que $l\left(t_{0}\right)=0$, y $t_{0}$ es etiquetado con la tesis del juego.

- Para cada $t \neq t_{0}$ hay un único $t^{\prime}$ tal que $t^{\prime} S t$.

- Para cada $t$ y $t^{\prime}$ en $T$, si $t S t^{\prime}$ entonces $l\left(t^{\prime}\right)=l(t)+1$.

- Dada la partida $\pi$ en $\mathcal{D}(A)$ tal que $p_{\pi}\left(M^{\prime}\right)=p_{\pi}(M)+1$ y $t, t^{\prime}$ respectivamente etiquetadas con $M$ y $M^{\prime}$, entoncest $S t^{\prime}$.

Una estrategia para un jugador $\mathrm{X}$ en $\mathcal{D}(A)$ es una función que asigna un movimiento $\mathrm{M}$ a cada partida no terminal $\pi$ con un movimiento $\mathrm{Y}$ como último miembro tal que, si extendemos $\pi$ con $M$ obtenemos una partida. Una estrategia de X es ganadora si jugando de acuerdo con ella nos lleva a una victoria de X sin importar cómo juegue $\mathrm{Y}$.

La forma extensiva de una estrategia $\sigma$ de $\mathrm{X}$ en $\mathcal{D}(A)$ es el fragmento de árbol $\mathrm{E}_{A, \mathrm{~s}}=\left(T_{\sigma}, l_{\sigma}, S_{\sigma}\right)$ de $\mathrm{E}_{A}$ tal que: 
i) la raíz de $\mathrm{E}_{A, \sigma}$ es la raíz de $\mathrm{E}_{A}$

ii) Dado el nodo $t$ en $\mathrm{E}_{A}$ etiquetado con un movimiento $\mathrm{X}$, tenemos que $t S_{\sigma} t$ ' sea cual fuere $t S t$.

iii) Dado el nodo $t$ en $\mathrm{E}_{A}$ etiquetado con un movimiento Yy con al menos un $t$ ' tal que $t S t$,', entonces hay una única $\sigma(t)$ en $T_{\sigma}$ donde $t S_{\sigma} \sigma(t)$ y $\sigma(t)$ es etiquetada con el movimiento de $\mathrm{X}$ prescrito por $\sigma$.

He aquí algunos ejemplos de resultados metalógicos obtenidos en la literatura reciente y que corresponden al nivelde las estrategias ${ }^{18}$.

- Estrategias de ganancia para $\mathrm{P}$ y hojas. Sea w una estrategia ganadora para $\mathrm{P}$ en $\mathcal{D}(A)$. Entonces, cada hoja en $\mathrm{E}_{A, \mathrm{w}}$ está etiquetada con una proposición elemental de $\mathrm{P}$.

- Determinación. Hay una estrategia ganadora para $\mathrm{X}$ en $\mathcal{D}(A)$ si y solo si no hay una estrategia para $\mathrm{Y}$ en $\mathcal{D}(A)$.

- Corrección y Completitud para tablas semánticas (también llamadas árboles semánticos). Considérese una tabla semántica de primer orden y una estrategia dialógica de primer orden. Hay una tabla cerrada para $A$ si y solo si existe una estrategia ganadora para $\mathrm{P}$ en $\mathcal{D}(A)$.

- Dado que las tablas semánticas (para lógica de primer orden) son correctas y completas respecto a una semántica modelo-teorética; se sigue que la existencia de una estrategia ganadora para $\mathrm{P}$ coincide con la validez. Es decir: Hay una estrategia ganadora para $\mathrm{P}$ en $\mathrm{D}(A)$ si y solo si $A$ es válida.

El hecho de que la existencia de una estrategia P-ganadora coincida con la validez (hay una estrategia P-ganadora en $\mathcal{D}(A)$ si y solo si $A$ es válida) se sigue de corrección (soundness) y completitud (completeness) del método de tableau con respecto a la semántica modelo-teorética.

\section{Referencias bibliográficas}

Abelardo (1141), Dialectica, en De Rijk, L.M., ed., (1956).

Ackrill, J. L. (ed. and trans.) (1963), Aristotle. Categories and De Interpretatione. Oxford: Clarendon Press.

Aristóteles (1982), “Categorías”, Tratados de lógica (Órganon) I. Madrid: Gredos. (1995), "Sobre la interpretación”, Tratados de lógica (Órganon) II. Madrid: Gredos.

18 Estos resultados están probados, junto con otros, en Clerbout (2013). 
Bencivenga, Ermanno (2002), "Free Logics", en D. Gabbay and F. Guenthner, eds., Handbook of Philosophical Logic, $2^{\text {nd }}$ edition, Vol. 5, Dordrecht: Kluwer, pp. 147-196.

Brouwer, L. E. J. (1907), “On the Foundations of Mathematics”, en L. E. J. Brouwer, Collected Works. Philosophy and Foundations of Mathematics I, ed. Arend Heyting (1975). Amsterdam: North-Holland Publishing Company; pp. 72-97.

(1908), "Específicamente The Unreliability of The Logical Principles", en L. E. J. Brouwer, Collected Works. Philosophy and Foundations of Mathematics I, ed. Arend Heyting (1975). Amsterdam: North-Holland Publishing Company; pp. 107-111.

Cantor, Georg (1874), "Über eine Eigenschaft des Inbegriffes aller reellen algebraischen Zahlen", Über unendliche, lineare Punktmannigfaltigkeiten. Arbeiten zur Mengenlehre aus den Jahren 1872-1884. Leipzig: BSB B. G. Teubner Verlagsgesellschaft.

Clerbout, N. (2013), "First-Order Dialogical games and Tableaux", Journal of Philosophical Logic. Online first Publication. DOI: 10.1007/s10992-0139289-z.

(2014a), Etude sur quelques sémantiques dialogiques. Concepts fondamentaux et éléments de metathéorie. London: College Publications.

De Rijk, L.M. (ed.) (1956), Dialectica: First Complete Edition of the Parisian Manuscript; with an introduction by L.M. De Rijk (Introduction CVI pages; Text pages 51-637).

Dedekind, D. (1872), Stetigkeit und irrationale Zahlen. Braunschweig: Friedrich Vieweg und Sohn.

(1888/1893), Was sind und was sollen die Zahlen? Braunschweig: Friedrich Vieweg und Sohn.

Dummett, M. (1977), Elements of Intuitionism. Oxford: Oxford University Press.

Fontaine, M., Redmond J., Rahman S. (2009), "Etre et Etrechoisi, Vers une logique dynamique de la fiction", dans page personnel Shahid Rahman: http://stl. recherche.univ-lille3.fr/textesenligne/etre_et_etre_choisi.pdf

Frege, Gottlob (1884), Die Grundlagen der Arithmetik. Eine logisch mathematische Untersuchung über den Begriff der Zahl. Breslau: Verlag von Wilhelm Koebner.

(1948), "Sense and Reference", The Philosophical Review, Vol. 57, No. 3 (May, 1948); pp. 209-230.

(1983), Nachgelassene Schriften, edición de H. Hermes, F. Kambartel y F. Kaulbach. Hamburg: Felix Meiner Verlag Hamburg.

(1993/1879), Begriffsschrift [und andere Aufsätze], reedición de Ignacio Angelelli. Hildesheim, Zürich and New York: Georg Olms Verlag.

(1879), Begriffsschrift, eine der arithmetischen nachgebildete Formelsprache des reinen Denkens, Halle a. S.: Louis Nebert.

Gamut, L. T. F. (2002), Introducción a la lógica. Buenos Aires: Eudeba. 
Gabriel, Gottfried et al. (1976), Gottlob Frege. Wissenschaftlicher Briefwechsel. Hamburg: Felix Meiner Verlag.

Goodman, Nelson (1949), “On Likeness of Meaning”, Analysis, Vol. 10, No. 1 (Oct., 1949); pp. 1-7.

Kant, Immanuel (1993), Crítica de la razón pura, trad. Pedro Ribas, 9a edición. Madrid: Alfaguara-Santillana.

Lambert, Karel (1960), "The Definition of E! in Free Logic", Abstracts: The International Congress for Logic, Methodology and Philosophy of Science. Stanford: Stanford University Press.

(1983), Meinong and the Principle of Independence. Cambridge: The University Press.

(1991), Philosophical Applications of Free Logic (editor and contributor). Oxford: Oxford University Press.

(2003), Free Logic: Selected Essays. Cambridge \& New York: Cambridge University Press. Press.

(2004), Free Logic. Selected Essays. Cambridge: Cambridge University

(1997), Free Logics: Their Foundations, Character, and Some Applications Thereof. ProPhil: Projekte zur Philosophie, Bd. 1. Sankt Augustin, Germany: Academia.

Lorenzen, P. (1955), Einführung in die operative Logik und Mathematik. Berlin, Göttingen and Heidelberg: Springer.

(1978), "Logik und Agon", Atti del XII Congresso Internazionale di Filosofia, Venezia, Sansoni, 1960: 187-194. Reimpreso en Lorenzen \& Lorenz (1978: 1-8).

Lorenzen, P. \& Lorenz, K. (1978), Dialogische Logik. Darmstadt: Wissenschaftliche Buchgesellschaft.

Martin-Löf, P. (1984), Intuitionistic Type Theory - Notes by Giovanni Sambin of a series of lectures given in Padua, June 1980. Naples: Bibliopolis.

(1996), "On the Meanings of the Logical Constants and the Justifications of the Logical Laws", Nordic Journal of Philosophical Logic, Vol.1.1; pp.1160.

Parsons, T. (2012), “The tradictional square of opposition”, Stanford Encyclopedia of Philosophy: https://plato.stanford.edu/entries/square/). Consulta: diciembre 2016.

Platón (1987), Gorgias, Diálogos II. Madrid: Editorial Gredos; pp. 8-145.

(1992), La República, Diálogos IV. Madrid: Editorial Gredos.

Priest, Graham (2005), Towards Non-Being. The logic and Metaphysics of Intentionality. Oxford: Clarendon Press. 
Rahman, S. (2001), “On Frege's Nightmare. A Combination of Intuitionistic, Free and Paraconsistent Logics”, en H. Wansing, ed., Essays on Non-Classical Logic, River Edge, New Jersey: World Scientific; pp. 61-85.

Rahman, S. \& Fontaine, M. (2010), "Fiction, Creation and Fictionality An Overview", Revue Methodos (CNRS, UMR 8163, STL). A paraître.

Rahman, S. \& Keiff, L. (2004), “On how to be a dialogician”, en D. Vanderveken, ed., Logic, Thought and Action, Dordrecht: Springer; pp. 359-408.

Redmond, J. (2010), Logique dynamique de la fiction. Pour une approche dialogique. London: College Publications.

Reicher, M. (2014), "Nonexistent Objects", Stanford Encyclopedia of Philosophy: https://plato.stanford.edu/entries/nonexistent-objects/. Consulta: diciembre 2016.

Russell, Bertrand (1905), “On Denoting”, Mind, 14: 479-493.

Stepanians, Markus (2007), Gottlob Frege: una introducción. Londres: College Publications.

Tarski, Alfred (1983), Logic, Semantics, Metamathematics, J. Corcoran, ed. Indianapolis: Hackett.

van Benthem, Johan (1994), "General Dynamics", What is a logical system? Dov Gabbay, ed. Oxford: Clarendon Press, coll. "Studies in Logic and Computation".

Wittgenstein, L. (1953), Philosophical Investigations (PI). G.E.M. Anscombe and R. Rhees, eds., G.E.M. Anscombe (trans.). Oxford: Blackwell. 